\title{
The Contribution of Optical Coherence Tomography in Neuromyelitis Optica Spectrum Disorders
}

\author{
Javier Mateo', Olivia Esteban', Mireya Martínez', Andrzej Grzybowski ${ }^{2,3 *}$ \\ and Francisco Javier Ascaso ${ }^{1,4}$ \\ 'Department of Ophthalmology, Hospital Clínico Universitario Lozano Blesa, Zaragoza, Spain, ${ }^{2}$ Department of \\ Ophthalmology, Poznan City Hospital, Poznan, Poland, ${ }^{3}$ Chair of Ophthalmology, University of Warmia and Mazury, Olsztyn, \\ Poland, ${ }^{4}$ Aragón Health Research Institute (IIS Aragón), Zaragoza, Spain
}

OPEN ACCESS

Edited by:

Elena H. Martínez-Lapiscina, Hospital Clínic de Barcelona, Spain

Reviewed by: Hanna Gwendolyn Zimmermann, Charité Universitätsmedizin Berlin, Germany Christian Cordano, University of California, San Francisco, United States Andrés Cruz-Herranz, University of California, San Francisco, United States Lisanne Balk,

VU University Medical Center Netherlands

*Correspondence: Andrzej Grzybowski ae.grzybowski@gmail.com

Specialty section:

This article was submitted to

Neuro-Ophthalmology,

a section of the journal

Frontiers in Neurology

Received: 24 May 2017 Accepted: 04 September 2017 Published: 29 September 2017

Citation:

Mateo J, Esteban O, Martínez M, Grzybowski A and Ascaso FJ (2017)

The Contribution of Optical Coherence Tomography in Neuromyelitis Optica Spectrum Disorders. Front. Neurol. 8:493. doi: 10.3389/fneur.2017.00493
Neuromyelitis optica spectrum disorders (NMOSD) comprises a group of central nervous system disorders of inflammatory autoimmune origin that mainly affect the optic nerves and the spinal cord and can cause severe visual and general disability. The clinical signs are similar to those of multiple sclerosis (MS), with the result that it is often difficult to differentiate between the two, thus leading to misdiagnosis. As the treatment and prognosis of NMOSD and MS are different, it is important to make an accurate and early diagnosis of NMOSD. Optical coherence tomography $(\mathrm{OCT})$ is a non-invasive technique that enables a quantitative study of the changes that the optic nerve and the macula undergo in several neurodegenerative diseases. Many studies have shown that some of these changes, such as retinal nerve fiber layer thinning or microcystic macular edema, can be related to alterations in the brain due to neurodegenerative disorders. The purpose of this mini-review is to show how OCT can be useful for the diagnosis of NMOSD and follow-up of affected patients, as well as for the differential diagnosis with MS.

Keywords: neuromyelitis optica, optical coherence tomography, optic neuritis, multiple sclerosis, autoimmune diseases, Devic disease

\section{INTRODUCTION}

Neuromyelitis optica spectrum disorders (NMOSD), traditionally known as neuromyelitis optica or Devic disease, comprise a group of uncommon central nervous system (CNS) disorders of inflammatory autoimmune origin that mainly affect the optic nerves and/or the spinal cord (1-4).

Neuromyelitis optica spectrum disorders were first reported in the nineteenth century (5). Patients suffering from NMOSD can develop optic neuritis $(\mathrm{ON})$ as well as transverse myelitis. ON, which is often the first manifestation of NMOSD, causes visual loss and painful eye movement, while myelitis can cause multiple alterations, such as sensory and movement disturbance, weakness, and loss of bowel and bladder control $(1,2,5)$. Although these are the main clinical manifestations of the disease, other areas of the CNS can be affected, including the brain stem, diencephalon, area postrema on the dorsal surface of the medulla oblongata, and cerebrum (6-9). Alterations of the CNS due to NMOSD often generate severe visual and systemic disability $(5,10)$.

The clinical signs and symptoms of NMOSD are similar to those of multiple sclerosis (MS), thus making it difficult to perform a correct differential diagnosis (DD) between these two entities. As the treatment and prognosis of NMOSD and MS are different, it is important to make an accurate and early diagnosis of NMOSD (10). In general, ON is more severe and recurrent and more often 
bilateral in NMOSD than in MS. In addition, visual impairment is more severe, and recovery is poorer in NMOSD (10-13). The finding of aquaporin-4 immunoglobulin G (AQP4-IgG) in blood considerably facilitates diagnosis, as it is present in most patients with NMOSD, but not in MS $(5,10,14,15)$. Magnetic resonance imaging (MRI) findings, such as optic chiasm involvement in ON or alteration of at least three contiguous segments of the spinal cord in transverse myelitis, are highly suggestive of NMOSD (16-18). A newer nomenclature of NMOSD is now being used to include patients with clinical syndromes and/or MRI findings with or without AQP4-IgG. ON is not always present in NMOSD. In addition, antibodies against myelin oligodendrocyte glycoprotein (MOG-IgG) are found in one-third of patients with AQP4-IgG-seronegative NMOSD. MOG-IgG antibodies are useful for the DD with MS. Furthermore, disease course is usually more favorable in patients with AQP4-IgG-seronegative and MOG-IgG-seropositive NMOSD (19, 20).

Optical coherence tomography (OCT) is a non-invasive imaging technique that was created in the 1990s and is currently used worldwide. It enables an in vivo quantitative and qualitative study of changes in the optic nerve and the macula $(10,21)$. It is fast, comfortable for the patient, and easy to perform and has, therefore, been used for several years to study many ophthalmic diseases, mainly glaucoma and macular disorders. In neuroophthalmology, OCT is considered a "window" to the CNS, and is widely used to study neurodegenerative diseases such as MS, NMOSD, Alzheimer's disease, and Parkinson's disease (10, 22, 23).

In this mini-review, we show how OCT can lead to a faster and more accurate diagnosis and follow-up of NMOSD and also how it can help to differentiate NMOSD from other neurodegenerative disorders, especially MS.

\section{METHODS}

We performed a literature search in PubMed in April 2017 using the terms "neuromyelitis optica" and "neuromyelitis optica spectrum disorders" combined with "optical coherence tomography" or "OCT." We looked for original case-control studies, case series, or cohort studies, as well as reviews on the topic "neurodegenerative disorders," "multiple sclerosis," "neuromyelitis optica," "optic neuritis," or "optic neuropathy." We also reviewed many of the references of the articles found.

\section{RESULTS}

The most usual finding when OCT is used in patients with NMOSD is thinning of the peripapillary retinal nerve fiber layer (pRNFL). NMOSD studies, some cross-sectional studies, and cohort and prospective longitudinal studies show a marked decrease in pRNFL thickness after ON $(11,15,23-27)$. As this finding is also observed in $\mathrm{ON}$ associated with other disorders, it does not facilitate the DD. However, many authors report that the decrease in pRNFL thickness and also in macular volume is more pronounced after ON in NMOSD than in MS (Table 1) $(11,15,28-34)$. This could be explained by the fact that episodes of ON in NMOSD tend to be more severe and recovery poorer than in MS (Figure 1A) $(11,29,30)$.
TABLE 1 | Average peripapillary retinal nerve fiber thickness (in micrometers) after optic neuritis in patients with neuromyelitis optica spectrum disorders (NMOSD), multiple sclerosis (MS), and healthy controls.

\begin{tabular}{llccc}
\hline & & NMOSD & MS & Controls \\
\hline Ratchford & Range & $31-99.4$ & $44.4-129.5$ & $80.8-128.1$ \\
et al. (28) & Mean \pm SD & $63.6 \pm 20.3$ & $88.3 \pm 16.5$ & $102.4 \pm 11.0$ \\
Schneider & Range & $28-93.4$ & $62.3-105.2$ & $80.7-122.3$ \\
et al. (29) & Mean \pm SD & $58.5 \pm 21.2$ & $85.3 \pm 13.3$ & $100.1 \pm 10.8$ \\
Manogaran & Mean \pm SD & $70.8 \pm 16.4$ & $81.0 \pm 12.8$ & $100.1 \pm 10.8$ \\
et al. (30) & & & & \\
Tian et al. (33) & Mean \pm SD & $63.94 \pm 11.86$ & $79.12 \pm 15.64$ & $112.01 \pm 10.93$ \\
Naismith & Mean \pm SD & $54.8 \pm 3.7$ & $76.5 \pm 2.4$ & \\
et al. (35) & & & & \\
Park et al. (36) & Mean \pm SD & $49.53 \pm 3.81$ & $70.09 \pm 5.78$ & $100.08 \pm 9.34$
\end{tabular}

When differences in thinning of pRNFL are compared between NMOSD and MS, the damage in NMOSD affects every quadrant, mainly the superior and inferior quadrant, while in MS, it is more severe in the temporal quadrant $(15,24,32,35)$. Some authors state that this alteration of the different sectors of the pRNFL is caused by damage to the small-diameter axons-located mainly in the temporal quadrant-in MS and a more diffuse injury in NMOSD $(15,24)$.

Another frequent alteration highlighted by OCT in NMOSD is the reduced thickness of the ganglion cell layer (GCL) in the macula $(10,11,15,33,36)$. This alteration has also been described in other neurodegenerative diseases, such as Alzheimer's disease and MS $(10,37)$. However, it seems that the decrease in the thickness of the GCL is more pronounced in NMOSD owing to intense inflammation and necrosis, with more prominent neuronal and axonal damage in NMOSD than in MS $(11,36)$. The more severe thinning of the superior quadrant of the GCL, which is similar to that observed in anterior ischemic optic neuropathy (AION), suggests vascular and ischemic damage to the optic nerve in NMOSD $(36,38)$. Recent investigations show foveal thinning in NMOSD patients with or without $\mathrm{ON}$, indicating damage to the retina, perhaps because Müller cells and retinal astrocytes expressing AQP4 are targeted in NMOSD, even when there is no history of ON (39-41).

In some cases of NMOSD, microcystic macular edema can affect the inner nuclear layer of the retina after $\mathrm{ON}$, a feature that has also been described in other conditions of the optic nerve, such as Leber hereditary optic neuropathy, AION, MS, compressive or traumatic optic neuropathy, hydrocephalus, and even glaucoma. The origin of this edema remains unclear, although the main hypotheses are trans-synaptic degeneration and vitreous traction $(15,21,24,42,43)$.

When ON is not present in NMOSD, there also seem to be differences between MS and NMOSD. While in MS there is significant subclinical thinning of the pRNFL, especially in the temporal quadrant, this damage does not occur or is very subtle in NMOSD without ON (Figure 1B) (15, 26-29). Nevertheless, there does seem to be a subclinical decrease in the thickness of the GCL and macular thickness in NMOSD without ON $(11,15,24,30,44,45)$. This might be caused by damage of the Müller cells in the retina (see above). These cells are rich in AQP4 channels, which are attacked in $\operatorname{NMOSD}(11,45)$. 


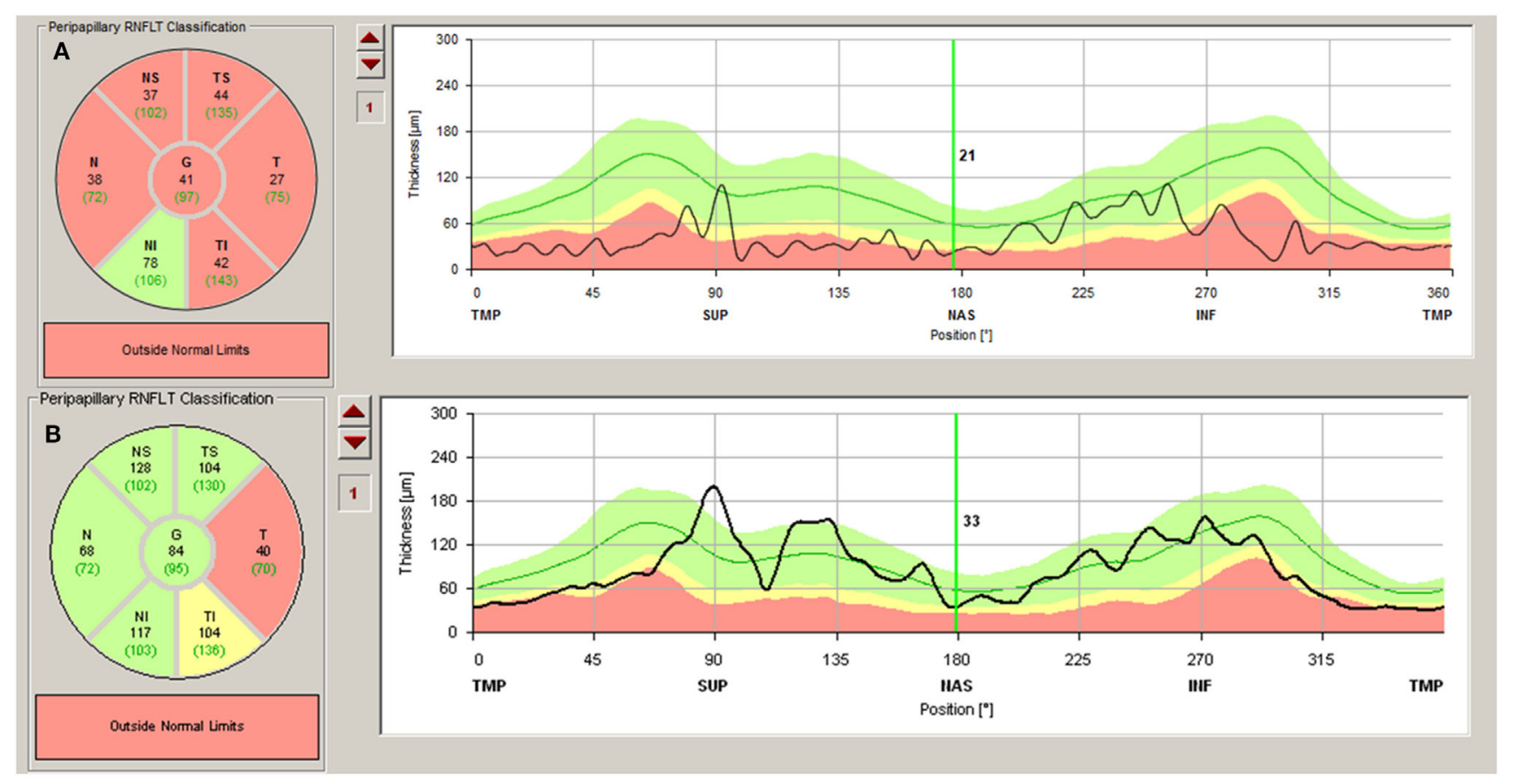

FIGURE 1 | (A) Severe atrophy of peripapillary retinal nerve fiber layer (pRNFL) after optic neuritis (ON) in a neuromyelitis optica spectrum disorders patient. (B) Atrophy of pRNFL temporal quadrant in a multiple sclerosis patient without history of ON.

A relationship can be observed between OCT changes and clinical features in NMOSD. For example, the decrease in pRNFL thickness and macular volume is apparently associated with the degree of visual disability in NMOSD, and the same can be observed with the decrease in the thickness of the GCL. Similar effects can be observed in MS $(10,15,24,33,46)$. Although while in MS there is a clear correlation between the reduction in pRNFL and GCL thickness and general disability, this correlation has not been reported as frequently in $\operatorname{NMOSD}(15,26,47-50)$.

\section{DISCUSSION}

Although OCT alone cannot confirm the DD between NMOSD and MS, it is highly accurate and plays a useful role in the followup of NMOSD.

The diagnostic criteria for NMOSD include clinical features (mainly ON and acute myelitis), the presence of AQP4-IgG in most patients, and characteristic MRI findings (3).

Owing to the similarities between NMOSD and MS, it is sometimes difficult to discriminate between both entities. Combination of OCT and the aforementioned diagnostic criteria can enable a more precise DD.

During the acute and early phase of $\mathrm{ON}$, when the optic nerve head is swollen and the pRNFL does not provide reliable data, OCT is particularly useful for assessment of the GCL, which is frequently more severely affected in NMOSD than in MS (33).

Other OCT changes that can facilitate the DD are thinning of the pRNFL and GCL and reduction in macular volume, which are usually more severe in NMOSD than in MS after ON $(11,15)$.
These changes, which predominantly affect the superior and inferior quadrants, are suggestive of NMOSD, while damage in the temporal quadrant points to MS $(15,24)$.

In cases of suspicion of NMOSD without ON, the thinning of the temporal quadrant of the pRNFL is characteristic of MS rather than NMOSD, since in the latter, pRNFL is only slightly affected or unaffected $(15,24)$.

Because of the relationship between damage to visual function and the decrease in pRNFL, macular volume, and the GCL, OCT could prove to be a valuable instrument for the follow-up of NMOSD $(10,15,24)$.

Further studies are necessary to provide more evidence and show us the extent of the usefulness of OCT for the diagnosis, study, and follow-up of NMOSD.

\section{AUTHOR CONTRIBUTIONS}

JM, OE, and MM: concept, design, collection of data, writing MS, and discussion. AG and FA: concept, design, and discussion.

\section{ACKNOWLEDGMENTS}

The authors would like to thank Bayer AG, for their support in the English editing and academic writing review.

\section{FUNDING}

The study was partly funded by Foundation for Ophthalmology Development, Poznan, Poland (AG). 


\section{REFERENCES}

1. Wingerchuk DM, Lennon VA, Lucchinetti CF, Pittock SJ, Weinshenker BG. The spectrum of neuromyelitis optica. Lancet Neurol (2007) 6:805-15. doi:10.1016/S1474-4422(07)70216-8

2. Wingerchuk DM, Hogancamp WF, O'Brien PC, Weinshenker BG. The clinical course of neuromyelitis optica (Devic's syndrome). Neurology (1999) 53:1107-14. doi:10.1212/WNL.53.5.1107

3. Wingerchuk DM, Banwell B, Bennett JL, Cabre P, Carroll W, Chitnis T, et al. International consensus diagnostic criteria for neuromyelitis optica spectrum disorders. Neurology (2015) 85:177-89. doi:10.1212/WNL. 0000000000001729

4. Jarius S, Wildemann B. The history of neuromyelitis optica. JNeuroinflammation (2013) 10:8. doi:10.1186/1742-2094-10-8

5. Morrow MJ, Wingerchuk D. Neuromyelitis optica. J Neuroophthalmol (2012) 32:154-66. doi:10.1097/WNO.0b013e31825662f1

6. Kremer L, Mealy M, Jacob A, Nakashima I, Cabre P, Bigi S, et al. Brainstem manifestations in neuromyelitis optica: a multicenter study of 258 patients. Mult Scler (2014) 20:843-7. doi:10.1177/1352458513507822

7. Poppe AY, Lapierre Y, Melançon D, Lowden D, Wardell L, Fullerton LM, et al. Neuromyelitis optica with hypothalamic involvement. Mult Scler (2005) 11:617-21. doi:10.1191/1352458505ms1200cr

8. Kim W, Kim SH, Lee SH, Li XF, Kim HJ. Brain abnormalities as an initial manifestation of neuromyelitis optica spectrum disorder. Mult Scler (2011) 17:1107-12. doi:10.1177/1352458511404917

9. Vernant JC, Cabre P, Smadja D, Merle H, Caubarrère I, Mikol J, et al. Recurrent optic neuromyelitis with endocrinopathies: a new syndrome. Neurology (1997) 48:58-64. doi:10.1212/WNL.48.1.58

10. Simao LM. The contribution of optic coherence tomography in neurodegenerative diseases. Curr Opin Ophthalmol (2013) 24:521-7. doi:10.1097/ ICU.0000000000000000

11. Manogaran P, Hanson JVM, Olbert ED, Egger C, Wicki C, Gerth-Kahlert C, et al. Optic coherence tomography and magnetic resonance imaging in multiple sclerosis and neuromyelitis optica spectrum disorder. Int J Mol Sci (2016) 17:1894. doi:10.3390/ijms17111894

12. Balcer LJ, Miller DH, Reingold SC, Cohen JA. Vision and vision-related outcome measures in multiple sclerosis. Brain (2015) 138:11-27. doi:10.1093/ brain/awu335

13. Fernandes DB, Ramos Rde I, Falcochio C, Apóstolos-Perreira S, Callegaro D, Monteiro ML. Comparison of visual acuity and automated perimetry findings in patients with neuromyelitis optica or multiple sclerosis after single or multiple attacks of optic neuritis. J Neuroophthalmol (2012) 32:102-6. doi:10.1097/ WNO.0b013e31823a9ebc

14. Lennon VA, Wingerchuk DM, Kryzer TJ, Pittock SJ, Lucchinetti CF, Fujihara K, et al. A serum autoantibody marker of neuromyelitis optica: distinction from multiple sclerosis. Lancet (2004) 364:2106-12. doi:10.1016/ S0140-6736(04)17551-X

15. Bennett JL, de Seze J, Lana-Peixoto M, Palace J, Waldman A, Schippling S, et al. Neuromyelitis optica and multiple sclerosis: seeing differences through optical coherence tomography. Mult Scler (2015) 21:678-88. doi:10.1177/ 1352458514567216

16. Huh SY, Min JH, Kim W, Kim SH, Kim HJ, Kim BJ, et al. The usefulness of brain MRI at onset in the differentiation of multiple sclerosis and seropositive neuromyelitis optica spectrum disorders. Mult Scler (2014) 20:695-704. doi:10.1177/1352458513506953

17. Yonezu T, Ito S, Mori M, Ogawa Y, Makino T, Uzawa A, et al. Bright spotty lesions on spinal magnetic resonance imaging differentiate neuromyelitis optica from multiple sclerosis. Mult Scler (2014) 20:331-7. doi:10.1177/ 1352458513495581

18. Pula JH, Kattah JC, Keung B, Wang H, Daily J. Longitudinally extensive optic neuritis in neuromyelitis optica spectrum disorder. J Neurol Sci (2014) 345:209-12. doi:10.1016/j.jns.2014.07.049

19. van Pelt ED, Wong YYM, Ketelslegers IA, Hamann D, Hintzen RQ. Neuromyelitis optica spectrum disorders: comparison of clinical and magnetic resonance imaging characteristics of AQP4-IgG versus MOG-IgG seropositive cases in the Netherlands. Eur J Ophthalmol (2016) 23:580-7. doi:10.1111/ene. 12898

20. Martínez-Lapiscina EH, Sepúlveda M, Torres-Torres R, Alba-Arbalat S, Llufriu S, Blanco Y, et al. Usefulness of optic coherence tomography to distinguish optic neuritis associated with AQP4 or MOG in neuromyelitis optica spectrum disorders. Ther Adv Neurol Disord (2016) 6:436-40. doi:10.1177/1756285616655264

21. Tawse KL, Hedges TR III, Gobuty M, Mendoza-Santiesteban C. Optical coherence tomography shows retinal abnormalities associated with optic nerve disease. Br JOphthalmol (2014) 98:ii30-3. doi:10.1136/ bjophthalmol-2013-304301

22. Galetta KM, Calabresi PA, Frohman EM, Balcer LJ. Optical coherence tomography (OCT): imaging the visual pathways as a model for neurodegeneration. Neurotherapeutics (2011) 8:117-32. doi:10.1007/s13311-010-0005-1

23. Jones-Odeh E, Hammond CJ. How strong is the relationship between glaucoma, the retinal nerve fibre layer, and neurodegenerative diseases such as Alzheimer's disease and multiple sclerosis? Eye (2015) 29:1270-84. doi:10.1038/eye.2015.158

24. Outteryck O, Majed B, Defoort-Dhellemmes S, Vermersch P, Zéphir H. A comparative optical coherence tomography study in neuromyelitis optica spectrum disorder and multiple sclerosis. Mult Scler (2015) 21:1781-93. doi: $10.1177 / 1352458515578888$

25. Merle H, Olindo S, Donnio A, Richer R, Smadja D, Cabre P. Retinal peripapillary nerve fiber layer thickness in neuromyelitis optica. Invest Ophthalmol Vis Sci (2008) 49:4412-7. doi:10.1167/iovs.08-1815

26. de Seze J, Blanc F, Jeanjean L, Zéphir H, Labauge P, Bouyon M, et al. Optical coherence tomography in neuromyelitis optica. Arch Neurol (2008) 65:920-3. doi:10.1001/archneur.65.7.920

27. Lange AP, Sadjadi R, Zhu F, et al. Spectral-domain optical coherence tomography of retinal nerve fiber layer thickness in NMO patients. J Neuroophthalmol (2013) 33:213-9. doi:10.1097/WNO.0b013e31829c510e

28. Ratchford JN, Quigg ME, Conger A, Frohman T, Frohman E, Balcer LJ, et al. Optical coherence tomography helps differentiate neuromyelitis optica and MS optic neuropathies. Neurology (2009) 73:302-8. doi:10.1212/ WNL.0b013e3181af78b8

29. Schneider E, Zimmermann H, Oberwahrenbrock T, Kaufhold F, Kadas EM, Petzold A, et al. Optical coherence tomography reveals distinct patterns of retinal damage in neuromyelitis optica and multiple sclerosis. PLoS One (2013) 8:e66151. doi:10.1371/journal.pone.0066151

30. Manogaran P, Vavasour IM, Lange AP, Zhao Y, McMullen K, Rauscher A, et al. Quantifying visual pathway axonal and myelin loss in multiple sclerosis and neuromyelitis optica. Neuroimage Clin (2016) 11:743-50. doi:10.1016/ j.nicl.2016.05.014

31. Monteiro MLR, Fernandes DB, Apóstolos-Pereira SL, Callegaro D. Quantification of retinal neural loss in patients with neuromyelitis optica and multiple sclerosis with or without optic neuritis using Fourier-Domain optic coherence tomography. Invest Ophthalmol Vis Sci (2012) 53:3959-66. doi:10.1167/iovs.11-9324

32. Noval S, Contreras I, Muñoz S, Oreja-Guevara C, Manzano B, Rebolleda G. Optical coherence tomography in multiple sclerosis and neuromyelitis optica: an update. Mult Scler Int (2011) 2011:472790. doi:10.1155/2011/ 472790

33. Tian G, Li Z, Zhao G, Feng C, Li M, Huang Y, et al. Evaluation of retinal nerve fiber layer and ganglion cell complex in patients with optic neuritis or neuromyelitis optica spectrum disorders using optical coherence tomography in a Chinese cohort. J Ophthalmol (2015) 2015:832784. doi:10.1155/2015/ 832784

34. Bichuetti DB, de Camargo AS, Falcao AB, Gonçalves FF, Tavares IM, de Oliveira EM. The retinal nerve fiber layer of patients with neuromyelitis optica and chronic relapsing optic neuritis is more severely damaged than patients with multiple sclerosis. JNeuroophthalmol (2013) 33:220-4 doi:10.1097/WNO.0b013e31829f39f1

35. Naismith RT, Tultman NT, Xu J, Klawiter EC, Shepherd J, Trinkaus K, et al. Optical coherence tomography differs in neuromyelitis optica compared with multiple sclerosis. Neurology (2009) 72:1077-82. doi:10.1212/01. wnl.0000345042.53843.d5

36. Park KA, Kim J, Oh SY. Analysis of spectral domain optical coherence tomography measurements in optic neuritis: differences in neuromyelitis optica, multiple sclerosis, isolated optic neuritis and normal healthy controls. Acta Ophthalmol (2014) 92:e57-65. doi:10.1111/aos.12215

37. Hinton DR, Sadun AA, Blanks JC, Miller CA. Optic-nerve degeneration in Alzheimer's disease. N Engl JMed (1986) 315:485-7. doi:10.1056/ NEJM198608213150804 
38. Contreras I, Noval S, Rebolleda G, Muñor-Negrete FJ. Follow-up of nonarteritic anterior optic neuropathy with optical coherence tomography. Ophthalmology (2007) 114:2338-44. doi:10.1016/j.ophtha.2007.05.042

39. Yamamura T, Nakashima I. Foveal thinning in neuromyelitis optica. A sign of retinal astrocitopathy? Neurol Neuroimmunol Neuroinflamm (2017) 4:e347. doi:10.1212/NXI.0000000000000347

40. Oertel FC, Kuchling J, Zimmerman H, Chien C, Schmidt F, Knier B, et al. Microstructural visual system changes in AQP4-antibody-seropositive NMSOD. Neurol Neuroimmunol Neuroinflamm (2017) 4:e334. doi:10.1212/ NXI.0000000000000334

41. Jeong IH, Kim HJ, Kim NH, Jeong KS, Park CY. Subclinical primary retinal pathology in neuromyelitis optica spectrum disorder. J Neurol (2016) 263:1343-8. doi:10.1007/s00415-016-8138-8

42. Gelfand JM, Cree BA, Nolan R, Arnow S, Green AJ. Microcystic inner nuclear layer abnormalities and neuromyelitis optica. JAMA Neurol (2013) 70:629-33. doi:10.1001/jamaneurol.2013.1832

43. Kaufhold F, Zimmermann H, Schneider E, Ruprecht K, Paul F, Oberwahrenbrock T, et al. Optic neuritis is associated with inner nuclear layer thickening and microcystic macular edema independently of multiple sclerosis. PLoS One (2013) 8:e71145. doi:10.1371/journal.pone. 0071145

44. Sotirchos ES, Saidha S, Byraiah G, Mealy MA, Ibrahim MA, Sepah YJ, et al. In vivo identification of morphologic retinal abnormalities in neuromyelitis optica. Neurology (2013) 80:1406-14. doi:10.1212/WNL. 0b013e31828c2f7a

45. Syc SB, Saidha S, Newsome SD, Ratchford JN, Levy M, Crainiceanu CM, et al. Optical coherence tomography segmentation reveals ganglion cell layer pathology after optic neuritis. Brain (2012) 135:521-33. doi:10.1093/brain/ awr264
46. Walter SD, Ishikawa H, Galetta KM, Sakai RE, Feller DJ, Henderson SB, et al. Ganglion cell loss in relation to visual disability in multiple sclerosis. Ophthalmology (2012) 119:1250-7. doi:10.1016/j.ophtha.2011.11.032

47. Sriram P, Wang C, Yiannikas C, Garrick R, Barnett M, Parrat J, et al. Relationship between optical coherence tomography and electrophysiology of the visual pathway in non-optic neuritis eyes of multiple sclerosis patients. PLoS One (2014) 9:e102546. doi:10.1371/journal.pone.0102546

48. García-Martín E, Polo V, Larrosa JM, Marques ML, Herrero R, Martín J, et al. Retinal layer segmentation in patients with multiple sclerosis using spectral domain optic coherence tomography. Ophthalmology (2014) 121:573-9. doi:10.1016/j.ophtha.2013.09.035

49. García-Martín E, Rodríguez-Mena D, Herrero R, Almarcegui C, Dolz I, Martín J, et al. Neuro-ophthalmologic evaluation, quality of life, and functional disability in patients with MS. Neurology (2013) 81:76-83. doi:10.1212/ WNL.0b013e318299ccd9

50. Pueyo V, Martin J, Fernandez J, Almarcegui C, Ara J, Egea C, et al. Axonal loss in the retinal fiber layer in patients with multiple sclerosis. Mult Scler (2008) 14:609-14. doi:10.1177/1352458507087326

Conflict of Interest Statement: The authors declare that the research was conducted in the absence of any commercial or financial relationships that could be construed as a potential conflict of interest.

Copyright (C) 2017 Mateo, Esteban, Martínez, Grzybowski and Ascaso. This is an open-access article distributed under the terms of the Creative Commons Attribution License (CC BY). The use, distribution or reproduction in other forums is permitted, provided the original author(s) or licensor are credited and that the original publication in this journal is cited, in accordance with accepted academic practice. No use distribution or reproduction is permitted which does not comply with these terms. 